\title{
MEROMORPHIC FUNCTIONS SHARING ONE VALUE
}

\author{
ABHIJIT BANERJEE
}

Received 13 April 2005 and in revised form 10 October 2005

We discuss the uniqueness problem of meromorphic functions sharing one value and obtain two theorems which improve a result of $\mathrm{Xu}$ and $\mathrm{Qu}$ and supplement some other results earlier given by Yang, Hua, and Lahiri.

\section{Introduction, definitions, and results}

Let $f$ and $g$ be two nonconstant meromorphic functions defined in the open complex plane $\mathbb{C}$. If for some $a \in \mathbb{C} \cup\{\infty\}, f-a$ and $g-a$ have the same set of zeros with the same multiplicities, we say that $f$ and $g$ share the value $a \mathrm{CM}$ (counting multiplicities), and if we do not consider the multiplicities, then $f$ and $g$ are said to share the value $a$ IM (ignoring multiplicities). We denote by $T(r)$ the maximum of $T(r, f)$ and $T(r, g)$. The notation $S(r)$ denotes any quantity satisfying $S(r)=o(T(r))$ as $r \rightarrow \infty$, outside a set of finite linear measure.

We use $I$ to denote any set of infinite linear measure of $0<r<\infty$.

Due to Nevanlinna [9], it is well known that if $f$ and $g$ share four distinct values CM, then $f$ is a Möbius transformation of $g$.

Yang and Hua showed that similar conclusions hold for certain types of differential polynomials when they share only one value. They proved the following result.

Theorem 1.1 [12]. Let $f$ and $g$ be two nonconstant meromorphic functions, $n \geq 11$ an integer, and $a \in \mathbb{C}-\{0\}$. If $f^{n} f^{\prime}$ and $g^{n} g^{\prime}$ share the value a $C M$, then either $f=d g$ for some $(n+1)$ th root of unity $d$ or $g(z)=c_{1} e^{c z}$ and $f(z)=c_{2} e^{-c z}$ where $c, c_{1}$, and $c_{2}$ are constants satisfying $\left(c_{1} c_{2}\right)^{n+1} c^{2}=-a^{2}$.

Corresponding to entire functions, $\mathrm{Xu}$ and $\mathrm{Qu}$ proved the following result.

TheOREM 1.2 [10]. Let $f$ and $g$ be two nonconstant entire functions, $n \geq 12$ an integer, and $a \in \mathbb{C}-\{0\}$. If $f^{n} f^{\prime}$ and $g^{n} g^{\prime}$ share the value a IM, then either $f=d g$ for some $(n+1)$ th root of unityd or $g(z)=c_{1} e^{c z}$ and $f(z)=c_{2} e^{-c z}$, where $c, c_{1}$, and $c_{2}$ are constants and satisfy $\left(c_{1} c_{2}\right)^{n+1} c^{2}=-a^{2}$.

To state the next result, we require the following definition. 
Definition $1.3[4,5]$. Let $k$ be a nonnegative integer or infinity. For $a \in \mathbb{C} \cup\{\infty\}$, denote by $E_{k}(a ; f)$ the set of all $a$-points of $f$, where an $a$-point of multiplicity $m$ is counted $m$ times if $m \leq k$ and $k+1$ times if $m>k$. If $E_{k}(a ; f)=E_{k}(a ; g)$, say that $f, g$ share the value $a$ with weight $k$.

The definition implies that if $f, g$ share a value $a$ with weight $k$, then $z_{0}$ is an $a$-point of $f$ with multiplicity $m(\leq k)$ if and only if it is an $a$-point of $g$ with multiplicity $m(\leq k)$ and $z_{0}$ is an $a$-point of $f$ with multiplicity $m(>k)$ if and only if it is an $a$-point of $g$ with multiplicity $n(>k)$, where $m$ is not necessarily equal to $n$.

We write $f, g$ share $(a, k)$ to mean that $f, g$ share the value $a$ with weight $k$. Since $E_{k}(a ; f)=E_{k}(a ; g)$ implies $E_{p}(a ; f)=E_{p}(a ; g)$ for any integer $p(0 \leq p<k)$, clearly if $f$, $g$ share $(a, k)$, then $f, g$ share $(a, p)$ for any integer $p, 0 \leq p<k$. Also we note that $f, g$ share a value $a$ IM or CM if and only if $f, g$ share $(a, 0)$ or $(a, \infty)$, respectively.

With the notion of weighted sharing of values improving Theorem 1.1 the following result is proved in [5].

Theorem 1.4 [5]. Let $f$ and $g$ be two nonconstant meromorphic functions, $n \geq 11$ an integer, and $a \in \mathbb{C}-\{0\}$. If $f^{n} f^{\prime}$ and $g^{n} g^{\prime}$ share $(a, 2)$, then either $f=d g$ for some $(n+1)$ th root of unityd or $g(z)=c_{1} e^{c z}$ and $f(z)=c_{2} e^{-c z}$, where $c, c_{1}$, and $c_{2}$ are constants satisfying $\left(c_{1} c_{2}\right)^{n+1} c^{2}=-a^{2}$.

Now one may ask the following questions which are the motivations of the paper.

(i) What happens if in Theorem 1.2 the two nonconstant entire functions $f$ and $g$ are replaced by two nonconstant meromorphic functions?

(ii) In Theorem 1.4, can the nature of sharing the value $a$ be further relaxed? In the paper, we investigate the solutions of the above questions. We now state the following two theorems which are the main results of the paper.

THEOREM 1.5. Let $f$ and $g$ be two nonconstant meromorphic functions such that $n>22-$ $[5 \Theta(\infty ; f)+5 \Theta(\infty ; g)+\min \{\Theta(\infty ; f), \Theta(\infty ; g)\}]$, where $n$ is an integer. If for $a \in \mathbb{C}-\{0\}$, $f^{n} f^{\prime}$ and $g^{n} g^{\prime}$ share $(a, 0)$, then either $f=d g$ for some $(n+1)$ th root of unity $d$ or $g(z)=$ $c_{1} e^{c z}$ and $f(z)=c_{2} e^{-c z}$, where $c, c_{1}$, and $c_{2}$ are constants satisfying $\left(c_{1} c_{2}\right)^{n+1} c^{2}=-a^{2}$.

THEOREM 1.6. Let $f$ and $g$ be two nonconstant meromorphic functions and $n>\max \{8,12-$ $\{3 \Theta(\infty ; f)+3 \Theta(\infty ; g)\}\}$ an integer. If for $a \in \mathbb{C}-\{0\}, f^{n} f^{\prime}$ and $g^{n} g^{\prime}$ share $(a, 1)$, then either $f=d g$ for some $(n+1)$ th root of unityd or $g(z)=c_{1} e^{c z}$ and $f(z)=c_{2} e^{-c z}$, where $c$, $c_{1}$, and $c_{2}$ are constants satisfying $\left(c_{1} c_{2}\right)^{n+1} c^{2}=-a^{2}$.

Remark 1.7. In Theorem 1.5 if we take $f$ and $g$ to be two nonconstant entire functions, then the theorem is true for an integer $n \geq 12$. So Theorem 1.5 improves Theorem 1.2.

Remark 1.8. In Theorem 1.6 if we take $f$ and $g$ to be two nonconstant entire functions, then the theorem is true for an integer $n \geq 7$.

Through the standard definitions and notations of the value distribution theory available in [2], we explain some definitions and notations which are used in the paper.

Definition 1.9 [3]. For $a \in \mathbb{C} \cup\{\infty\}$, denote by $N(r, a ; f \mid=1)$ the counting function of simple $a$-points of $f$. For a positive integer $m$, denote by $N(r, a ; f \mid \leq m)(N(r, a ; f \mid \geq m))$ 
the counting function of those $a$-points of $f$ whose multiplicities are not greater (less) than $m$ where each $a$-point is counted according to its multiplicity.

$\bar{N}(r, a ; f \mid \leq m)(\bar{N}(r, a ; f \mid \geq m))$ are defined similarly, where in counting the $a$-points of $f$ we ignore the multiplicities.

Also $N(r, a ; f \mid<m), N(r, a ; f \mid>m), \bar{N}(r, a ; f \mid<m)$ and $\bar{N}(r, a ; f \mid>m)$ are defined analogously.

Definition 1.10 [5]. Denote by $N_{2}(r, a ; f)$ the $\operatorname{sum} \bar{N}(r, a ; f)+\bar{N}(r, a ; f \mid \geq 2)$.

Definition $1.11[1,15,16]$. Let $f$ and $g$ be two nonconstant meromorphic functions such that $f$ and $g$ share the value 1 IM. Let $z_{0}$ be a 1-point of $f$ with multiplicity $p$, a 1-point of $g$ with multiplicity $q$. Denote by $\bar{N}_{L}(r, 1 ; f)$ the counting function of those 1-points of $f$ and $g$ where $p>q$, denote by $N_{E}^{1)}(r, 1 ; f)$ the counting function of those 1-points of $f$ and $g$ where $p=q=1$, and denote by $\bar{N}_{E}^{(2}(r, 1 ; f)$ the counting function of those 1-points of $f$ and $g$ where $p=q \geq 2$, each point in these counting functions is counted only once. In the same way, one can define $\bar{N}_{L}(r, 1 ; g), N_{E}^{1)}(r, 1 ; g), \bar{N}_{E}^{(2}(r, 1 ; g)$.

Definition 1.12 (cf. [1]). Let $k$ be a positive integer. Let $f$ and $g$ be two nonconstant meromorphic functions such that $f$ and $g$ share the value 1 IM. Let $z_{0}$ be a 1-point of $f$ with multiplicity $p$, and a 1-point of $g$ with multiplicity $q$. Denote by $\bar{N}_{f>k}(r, 1 ; g)$ the reduced counting function of those 1 -points of $f$ and $g$ such that $p>q=k \cdot \bar{N}_{g>k}(r, 1 ; f)$ is defined analogously.

Definition $1.13[4,5]$. Let $f, g$ share a value IM. Denote by $\bar{N}_{*}(r, a ; f, g)$ the reduced counting function of those $a$-points of $f$ whose multiplicities differ from the multiplicities of the corresponding $a$-points of $g$.

Clearly $\bar{N}_{*}(r, a ; f, g) \equiv \bar{N}_{*}(r, a ; g, f)$ and $\bar{N}_{*}(r, a ; f, g)=\bar{N}_{L}(r, a ; f)+\bar{N}_{L}(r, a ; g)$.

Definition 1.14 [6]. Let $a, b \in \mathbb{C} \cup\{\infty\}$. Denote by $N(r, a ; f \mid g=b)$ the counting function of those $a$-points of $f$, counted according to multiplicity, which are the $b$-points of $g$.

Definition 1.15 [6]. Let $a, b \in \mathbb{C} \cup\{\infty\}$. Denote by $N(r, a ; f \mid g \neq b)$ the counting function of those $a$-points of $f$, counted according to multiplicity, which are not the $b$-points of $g$.

\section{Lemmas}

In this section, we present some lemmas which will be needed in the sequel. Let $f, g, F$, $G$ be four nonconstant meromorphic functions. Henceforth, we will denote by $h$ and $H$ the following two functions:

$$
\begin{gathered}
h=\left(\frac{f^{\prime \prime}}{f^{\prime}}-\frac{2 f^{\prime}}{f-1}\right)-\left(\frac{g^{\prime \prime}}{g^{\prime}}-\frac{2 g^{\prime}}{g-1}\right), \\
H=\left(\frac{F^{\prime \prime \prime}}{F^{\prime \prime}}-\frac{2 F^{\prime \prime}}{F^{\prime}-1}\right)-\left(\frac{G^{\prime \prime \prime}}{G^{\prime \prime}}-\frac{2 G^{\prime \prime}}{G^{\prime}-1}\right) .
\end{gathered}
$$

Lemma $2.1[15,16]$. If $f, g$ are two nonconstant meromorphic functions such that they share $(1,0)$ and $h \neq \equiv$, then

$$
N_{E}^{1)}(r, 1 ; f) \leq N(r, h)+S(r, f)+S(r, g) .
$$


Lemma 2.2 [7]. If $N\left(r, 0 ; f^{(k)} \mid f \neq 0\right)$ denotes the counting function of those zeros of $f^{(k)}$ which are not the zeros of $f$, where a zero of $f^{(k)}$ is counted according to its multiplicity, then

$$
N\left(r, 0 ; f^{(k)} \mid f \neq 0\right) \leq k \bar{N}(r, \infty ; f)+N(r, 0 ; f \mid<k)+k \bar{N}(r, 0 ; f \mid \geq k)+S(r, f) .
$$

Lemma 2.3. Let $f$ and $g$ be two nonconstant meromorphic functions sharing $(1,0)$. Then

$$
\begin{aligned}
& \bar{N}_{L}(r, 1 ; f)+2 \bar{N}_{L}(r, 1 ; g)+\bar{N}_{E}^{(2}(r, 1 ; f)-\bar{N}_{f>1}(r, 1 ; g)-\bar{N}_{g>1}(r, 1 ; f) \\
& \quad \leq N(r, 1 ; g)-\bar{N}(r, 1 ; g) .
\end{aligned}
$$

Proof. Let $z_{0}$ be a 1-point of $f$ of multiplicity $p$ a 1-point of $g$ of multiplicity $q$. We denote by $N_{1}(r), N_{2}(r)$, and $N_{3}(r)$ the counting functions of those 1-points of $f$ and $g$ when $1 \leq q<p, 2 \leq q=p$ and $p<q$, respectively, where in the first counting function each point is counted $q-1$ times and in the remaining two counting functions each point is counted $q-2$ times.

Since $f, g$ share $(1,0)$, we note that a simple 1-point of $g$ is either a simple 1-point of $f$ or a 1 -point of $f$ with multiplicity $\geq 2$. So we can write

$$
N(r, 1 ; g)-\bar{N}(r, 1 ; g)=\bar{N}_{E}^{(2}(r, 1 ; f)+\bar{N}_{L}(r, 1 ; g)+N_{1}(r)+N_{2}(r)+N_{3}(r)
$$

Also we note that

$$
\begin{aligned}
& N_{1}(r) \geq \bar{N}_{L}(r, 1 ; f)-\bar{N}_{f>1}(r, 1 ; g), \\
& N_{2}(r) \geq \bar{N}_{E}^{(2}(r, 1 ; f)-\bar{N}(r, 1 ; f, g \mid=2), \\
& N_{3}(r) \geq \bar{N}_{L}(r, 1 ; g)-\bar{N}_{g>1}(r, 1 ; f),
\end{aligned}
$$

where by $\bar{N}(r, 1 ; f, g \mid=2)$ we mean the reduced counting functions of 1-points of $f$ and $g$ with multiplicities two for each one.

Using (2.6)-(2.8) in (2.5), we deduce that

$$
\begin{aligned}
& N(r, 1 ; g)-\bar{N}(r, 1 ; g) \\
& \quad \begin{array}{l}
\quad \bar{N}_{L}(r, 1 ; f)+2 \bar{N}_{L}(r, 1 ; g)+2 \bar{N}_{E}^{(2}(r, 1 ; f) \\
\quad-\bar{N}(r, 1 ; f, g \mid=2)-\bar{N}_{f>1}(r, 1 ; g)-\bar{N}_{g>1}(r, 1 ; f) .
\end{array}
\end{aligned}
$$

Now (i) follows from (2.9). This proves the lemma.

Lemma 2.4 [1]. If $f, g$ are two nonconstant meromorphic functions such that they share $(1,1)$, then

$$
2 \bar{N}_{L}(r, 1 ; f)+2 \bar{N}_{L}(r, 1 ; g)+\bar{N}_{E}^{(2}(r, 1 ; f)-\bar{N}_{f>2}(r, 1 ; g) \leq N(r, 1 ; g)-\bar{N}(r, 1 ; g) .
$$


Lemma 2.5. Let $f, g$ share $(1,0)$ and $h \neq \equiv$, then

$$
\begin{aligned}
N(r, h) \leq & \bar{N}(r, 0 ; f \mid \geq 2)+\bar{N}(r, 0 ; g \mid \geq 2)+\bar{N}(r, \infty ; f \mid \geq 2) \\
& +\bar{N}(r, \infty ; g \mid \geq 2)+\bar{N}_{*}(r, 1 ; f, g)+\bar{N}_{0}\left(r, 0 ; f^{\prime}\right)+\bar{N}_{0}\left(r, 0 ; g^{\prime}\right)
\end{aligned}
$$

where $\bar{N}_{0}\left(r, 0 ; f^{\prime}\right)$ is the reduced counting function of those zeros of $f^{\prime}$ which are not the zeros of $f(f-1)$ and $\bar{N}_{0}\left(r, 0 ; g^{\prime}\right)$ is similarly defined.

Proof. We can easily verify that possible poles of $h$ occur at (i) multiple zeros of $f$ and $g$, (ii) multiple poles of $f$ and $g$, (iii) those 1-points of $f$ and $g$ whose multiplicities are distinct from the multiplicities of the corresponding 1-points of $g$ and $f$, respectively, (iv) zeros of $f^{\prime}$ which are not the zeros of $f(f-1)$ and $(\mathrm{v})$ zeros of $g^{\prime}$ which are not zeros of $g(g-1)$.

Since $h$ has only simple poles, the lemma follows from above. This proves the lemma.

Lemma 2.6 [15]. Let $f, g$ share $(1,0)$. Then

$$
\bar{N}_{L}(r, 1 ; f) \leq \bar{N}(r, 0 ; f)+\bar{N}(r, \infty ; f)+S(r) .
$$

Lemma 2.7. Let $f$, $g$ share $(1,0)$. Then

(i) $\bar{N}_{f>1}(r, 1 ; g) \leq \bar{N}(r, 0 ; f)+\bar{N}(r, \infty ; f)-N_{0}\left(r, 0 ; f^{\prime}\right)+S(r, f)$,

(ii) $\bar{N}_{g>1}(r, 1 ; f) \leq \bar{N}(r, 0 ; g)+\bar{N}(r, \infty ; g)-N_{0}\left(r, 0 ; f^{\prime}\right)+S(r, g)$.

Proof. We prove (i) because (ii) can be proved in a similar manner.

Using Lemma 2.2, we obtain

$$
\begin{aligned}
\bar{N}_{f>1}(r, 1 ; g) & \leq \bar{N}(r, 1 ; f \mid \geq 2) \\
& \leq N\left(r, 0 ; f^{\prime} \mid f=1\right) \\
& \leq N\left(r, 0 ; f^{\prime} \mid f \neq 0\right)-N_{0}\left(r, 0 ; f^{\prime}\right) \\
& \leq \bar{N}(r, 0 ; f)+\bar{N}(r, \infty ; f)-N_{0}\left(r, 0 ; f^{\prime}\right)+S(r, f) .
\end{aligned}
$$

Lemma 2.8. Let $f, g$ share $(1,1)$. Then

$$
\bar{N}_{f>2}(r, 1 ; g) \leq \frac{1}{2} \bar{N}(r, 0 ; f)+\frac{1}{2} \bar{N}(r, \infty ; f)-\frac{1}{2} N_{0}\left(r, 0 ; f^{\prime}\right)+S(r, f) .
$$

Proof. Using Lemma 2.2, we get

$$
\begin{aligned}
\bar{N}_{f>2}(r, 1 ; g) & \leq \bar{N}(r, 1 ; f \mid \geq 3) \\
& \leq \frac{1}{2} N\left(r, 0 ; f^{\prime} \mid f=1\right) \\
& \leq \frac{1}{2} N\left(r, 0 ; f^{\prime} \mid f \neq 0\right)-\frac{1}{2} N_{0}\left(r, 0 ; f^{\prime}\right) \\
& \leq \frac{1}{2} \bar{N}(r, 0 ; f)+\frac{1}{2} \bar{N}(r, \infty ; f)-\frac{1}{2} N_{0}\left(r, 0 ; f^{\prime}\right)+S(r, f) .
\end{aligned}
$$


3592 Meromorphic functions sharing one value

LeMma 2.9 [14]. If $h \equiv 0$ and

$$
\limsup _{r \rightarrow \infty} \frac{\bar{N}(r, 0 ; f)+\bar{N}(r, \infty ; f)+\bar{N}(r, 0 ; g)+\bar{N}(r, \infty ; g)}{T(r)}<1, \quad r \in I,
$$

where $T(r)=\max \{T(r, f), T(r, g)\}$, then $f \equiv g$ or $f \cdot g \equiv 1$.

LEMma 2.10 (cf. $[8,11]$ ). Let $f$ be a nonconstant meromorphic function and $P(f)=a_{0}+$ $a_{1} f+a_{2} f^{2}+\cdots+a_{n} f^{n}$, where $a_{0}, a_{1}, a_{2}, \ldots, a_{n}$ are constants and $a_{n} \neq 0$. Then $T(r, P(f))=$ $n T(r, f)+O(1)$.

LEMMA 2.11. Let $f$ be a nonconstant meromorphic function and $F=f^{n+1} / a(n+1), n$ being a positive integer. Then

$$
T(r, F) \leq T\left(r, F^{\prime}\right)+N(r, 0 ; f)-N\left(r, 0 ; f^{\prime}\right)+S(r, f) .
$$

Proof. By the first fundamental theorem and Milloux theorem, we get

$$
m\left(r, \frac{1}{F}\right) \leq m\left(r, \frac{F^{\prime}}{F}\right)+m\left(r, \frac{1}{F^{\prime}}\right)
$$

that is,

$$
N(r, 0 ; F)+m(r, 0 ; F) \leq N(r, 0 ; F)+m\left(r, 0 ; F^{\prime}\right)+S(r, F),
$$

that is,

$$
T(r, F) \leq T\left(r, F^{\prime}\right)+N(r, 0 ; F)-N\left(r, 0 ; F^{\prime}\right)+S(r, F) .
$$

Since $N(r, 0 ; F)=(n+1) N(r, 0 ; f)$ and $N\left(r, 0 ; F^{\prime}\right)=n N(r, 0 ; f)+N\left(r, 0 ; f^{\prime}\right)$ and by Lemma 2.10, $S(r, F)=S(r, f)$, then the lemma follows from (2.20). This proves the lemma.

Lemma 2.12. Let $f, g$ be two nonconstant meromorphic functions and $F=f^{n+1} / a(n+1)$, $G=g^{n+1} / a(n+1)$, where $n(>2)$ is an integer. Then $F^{\prime} \equiv G^{\prime}$ implies $F \equiv G$.

Proof. $F^{\prime} \equiv G^{\prime}$ then $F=G+c$ where $c$ is a constant. If possible, let $c \neq 0$. Then by the second fundamental theorem and Lemma 2.10, we get

$$
\begin{aligned}
(n+1) T(r, f) & \leq \bar{N}(r, \infty ; F)+\bar{N}(r, 0 ; F)+\bar{N}(r, c ; F)+S(r, F) \\
& =\bar{N}(r, \infty ; f)+\bar{N}(r, 0 ; f)+\bar{N}(r, 0 ; g)+S(r, f) \\
& \leq 2 T(r, f)+T(r, g)+S(r, f) \\
& \leq 3 T(r)+S(r) .
\end{aligned}
$$

In a similar manner, we get

$$
(n+1) T(r, g) \leq 3 T(r)+S(r) \text {. }
$$


This shows that

$$
(n-2) T(r) \leq S(r)
$$

which is a contradiction for $n>2$. This proves the lemma.

Lemma 2.13 [12]. Let $f, g$ be two nonconstant meromorphic functions and $n>6$. If $f^{n} f^{\prime} g^{n} g^{\prime}=1$, then $g=c_{1} e^{c z}, f=c_{2} e^{-c z}$, where $c, c_{1}, c_{2}$ are constants and $\left(c_{1} c_{2}\right)^{n+1} c^{2}=-1$.

LemMa 2.14. Let $f, g$ be two nonconstant meromorphic functions such that they share $(1,0)$ and $h \equiv 0$. Then

$$
\begin{aligned}
T(r, f) \leq & N_{2}(r, 0 ; f)+N_{2}(r, \infty ; f)+N_{2}(r, 0 ; g)+N_{2}(r, \infty ; g)+2 \bar{N}(r, 0 ; f) \\
& +2 \bar{N}(r, \infty ; f)+\bar{N}(r, 0 ; g)+\bar{N}(r, \infty ; g)+S(r, f)+S(r, g) .
\end{aligned}
$$

Proof. By the second fundamental theorem, we get

$$
\begin{aligned}
T(r, f) & +T(r, g) \\
\leq & \bar{N}(r, 0 ; f)+\bar{N}(r, \infty ; f)+\bar{N}(r, 0 ; g)+\bar{N}(r, \infty ; g)+\bar{N}(r, 1 ; f) \\
& +\bar{N}(r, 1 ; g)-N_{0}\left(r, 0 ; f^{\prime}\right)-N_{0}\left(r, 0 ; g^{\prime}\right)+S(r, f)+S(r, g) .
\end{aligned}
$$

By Lemmas 2.1, 2.3, and 2.5, we get

$$
\begin{aligned}
& \bar{N}(r, 1 ; f)+\bar{N}(r, 1 ; g) \\
& \leq N_{E}^{1)}(r, 1 ; f)+\bar{N}_{L}(r, 1 ; f)+\bar{N}_{L}(r, 1 ; g)+\bar{N}_{E}^{(2}(r, 1 ; f)+\bar{N}(r, 1 ; g) \\
& \leq N_{E}^{1)}(r, 1 ; f)+N(r, 1 ; g)-\bar{N}_{L}(r, 1 ; g)+\bar{N}_{f>1}(r, 1 ; g)+\bar{N}_{g>1}(r, 1 ; f) \\
& \leq \bar{N}(r, 0 ; f \mid \geq 2)+\bar{N}(r, 0 ; g \mid \geq 2)+\bar{N}(r, \infty ; f \mid \geq 2)+\bar{N}(r, \infty ; g \mid \geq 2) \\
&+\bar{N}_{*}(r, 1 ; f, g)+T(r, g)-m(r, 1 ; g)+O(1)-\bar{N}_{L}(r, 1 ; g)+\bar{N}_{f>1}(r, 1 ; g) \\
&+\bar{N}_{g>1}(r, 1 ; f)+\bar{N}_{0}\left(r, 0 ; f^{\prime}\right)+\bar{N}_{0}\left(r, 0 ; g^{\prime}\right)+S(r, f)+S(r, g) .
\end{aligned}
$$

Since $\bar{N}_{*}(r, 1 ; f, g)=\bar{N}_{L}(r, 1 ; f)+\bar{N}_{L}(r, 1 ; g)$, by Lemmas 2.6 and 2.7 , we get from $(2.25)$ and (2.26) in view of Definition 1.10 that

$$
\begin{aligned}
T(r, f) \leq & N_{2}(r, 0 ; f)+N_{2}(r, \infty ; f)+N_{2}(r, 0 ; g)+N_{2}(r, \infty ; g)+2 \bar{N}(r, 0 ; f) \\
& +2 \bar{N}(r, \infty ; f)+\bar{N}(r, 0 ; g)+\bar{N}(r, \infty ; g)+S(r, f)+S(r, g) .
\end{aligned}
$$

Lemma 2.15. Let $f, g$ be two nonconstant meromorphic functions such that they share $(1,1)$ and $h \not \equiv$. Then

$$
\begin{aligned}
T(r, f) \leq & N_{2}(r, 0 ; f)+N_{2}(r, \infty ; f)+N_{2}(r, 0 ; g)+N_{2}(r, \infty ; g) \\
& +\frac{1}{2} \bar{N}(r, 0 ; f)+\frac{1}{2} \bar{N}(r, \infty ; f)+S(r, f)+S(r, g) .
\end{aligned}
$$


3594 Meromorphic functions sharing one value

Proof. By the second fundamental theorem, we get

$$
\begin{aligned}
T(r, f) & +T(r, g) \\
\leq & \bar{N}(r, 0 ; f)+\bar{N}(r, \infty ; f)+\bar{N}(r, 0 ; g)+\bar{N}(r, \infty ; g)+\bar{N}(r, 1 ; f) \\
& +\bar{N}(r, 1 ; g)-N_{0}\left(r, 0 ; f^{\prime}\right)-N_{0}\left(r, 0 ; g^{\prime}\right)+S(r, f)+S(r, g) .
\end{aligned}
$$

Since $f, g$ share $(1,1), N_{E}^{1)}(r, 1 ; f)=N(r, 1 ; f \mid=1)$. So using Lemmas $2.1,2.4,2.5$, and 2.8 , we get

$$
\begin{aligned}
& \bar{N}(r, 1 ; f)+\bar{N}(r, 1 ; g) \\
& \leq N(r, 1 ; f \mid=1)+\bar{N}_{L}(r, 1 ; f)+\bar{N}_{L}(r, 1 ; g)+\bar{N}_{E}^{(2}(r, 1 ; f)+\bar{N}(r, 1 ; g) \\
& \leq N(r, 1 ; f \mid=1)+N(r, 1 ; g)-\bar{N}_{L}(r, 1 ; f)-\bar{N}_{L}(r, 1 ; g)+\bar{N}_{f>2}(r, 1 ; g) \\
& \leq \bar{N}(r, 0 ; f \mid \geq 2)+\bar{N}(r, 0 ; g \mid \geq 2)+\bar{N}(r, \infty ; f \mid \geq 2)+\bar{N}(r, \infty ; g \mid \geq 2) \\
&+\bar{N}_{*}(r, 1 ; f, g)+T(r, g)-m(r, 1 ; g)+O(1)-\bar{N}_{L}(r, 1 ; f)-\bar{N}_{L}(r, 1 ; g) \\
&+\frac{1}{2} \bar{N}(r, 0 ; f)+\frac{1}{2} \bar{N}(r, \infty ; f)+\bar{N}_{0}\left(r, 0 ; f^{\prime}\right)+\bar{N}_{0}\left(r, 0 ; g^{\prime}\right)+S(r, f)+S(r, g) .
\end{aligned}
$$

From (2.29) and (2.30), we obtain in view of Definition 1.10 that

$$
\begin{aligned}
T(r, f) \leq & N_{2}(r, 0 ; f)+N_{2}(r, \infty ; f)+N_{2}(r, 0 ; g)+N_{2}(r, \infty ; g) \\
& +\frac{1}{2} \bar{N}(r, 0 ; f)+\frac{1}{2} \bar{N}(r, \infty ; f)+S(r, f)+S(r, g) .
\end{aligned}
$$

This proves the lemma.

LemMa 2.16 [13]. Let $f$ be a nonconstant meromorphic function. Then

$$
N\left(r, 0 ; f^{(k)}\right) \leq k \bar{N}(r, \infty ; f)+N(r, 0 ; f)+S(r, f) .
$$

\section{Proofs of the theorems}

Proof of Theorem 1.5. Let $F=f^{n+1} / a(n+1)$ and $G=g^{n+1} / a(n+1)$. Then $F^{\prime}=f^{n} f^{\prime} / a$ and $G^{\prime}=g^{n} g^{\prime} / a$. Since $f^{n} f^{\prime}$ and $g^{n} g^{\prime}$ share $(a, 0)$, it follows that $F^{\prime}, G^{\prime}$ share $(1,0)$. If possible, we suppose that $H \not \equiv 0$. Then by Lemma 2.14, we obtain

$$
\begin{aligned}
T\left(r, F^{\prime}\right) \leq & N_{2}\left(r, 0 ; F^{\prime}\right)+N_{2}\left(r, \infty ; F^{\prime}\right)+N_{2}\left(r, 0 ; G^{\prime}\right)+N_{2}\left(r, \infty ; G^{\prime}\right)+2 \bar{N}\left(r, 0 ; F^{\prime}\right) \\
& +2 \bar{N}\left(r, \infty ; F^{\prime}\right)+\bar{N}\left(r, 0 ; G^{\prime}\right)+\bar{N}\left(r, \infty ; G^{\prime}\right)+S\left(r, F^{\prime}\right)+S\left(r, G^{\prime}\right) .
\end{aligned}
$$

We see that

$$
\begin{aligned}
N_{2}\left(r, 0 ; F^{\prime}\right)+N_{2}\left(r, \infty ; F^{\prime}\right) & \leq 2 \bar{N}(r, 0 ; f)+N\left(r, 0 ; f^{\prime}\right)+2 \bar{N}(r, \infty ; f), \\
N_{2}\left(r, 0 ; G^{\prime}\right)+N_{2}\left(r, \infty ; G^{\prime}\right) & \leq 2 \bar{N}(r, 0 ; g)+N\left(r, 0 ; g^{\prime}\right)+2 \bar{N}(r, \infty ; g), \\
2 \bar{N}\left(r, 0 ; F^{\prime}\right)+2 \bar{N}\left(r, \infty ; F^{\prime}\right) & \leq 2 \bar{N}(r, 0 ; f)+2 N\left(r, 0 ; f^{\prime}\right)+2 \bar{N}(r, \infty ; f), \\
\bar{N}\left(r, 0 ; G^{\prime}\right)+\bar{N}\left(r, \infty ; G^{\prime}\right) & \leq \bar{N}(r, 0 ; g)+N\left(r, 0 ; g^{\prime}\right)+\bar{N}(r, \infty ; g) .
\end{aligned}
$$


Also by Lemma 2.10, we get

$$
\begin{aligned}
& T\left(r, F^{\prime}\right) \leq 2 T(r, F)+S(r, F)=2(n+1) T(r, f)+S(r, f), \\
& T\left(r, G^{\prime}\right) \leq 2 T(r, G)+S(r, G)=2(n+1) T(r, g)+S(r, g) .
\end{aligned}
$$

So $S\left(r, F^{\prime}\right)=S(r, f)$ and $S\left(r, G^{\prime}\right)=S(r, g)$. So by Lemmas 2.11 and 2.16, we get from (3.1) for $\varepsilon(>0)$ that

$$
\begin{aligned}
T(r, F) \leq & T\left(r, F^{\prime}\right)+N(r, 0 ; f)-N\left(r, 0 ; f^{\prime}\right)+S(r, f) \\
\leq & 4 \bar{N}(r, 0 ; f)+N(r, 0 ; f)+3 \bar{N}(r, 0 ; g)+4 \bar{N}(r, \infty ; f) \\
& +3 \bar{N}(r, \infty ; g)+2 N\left(r, 0 ; f^{\prime}\right)+2 N\left(r, 0 ; g^{\prime}\right)+S(r, f)+S(r, g) \\
\leq & 7 T(r, f)+5 T(r, g)+(6-6 \Theta(\infty ; f)+\varepsilon) T(r, f) \\
& +(5-5 \Theta(\infty ; g)+\varepsilon) T(r, g)+S(r, f)+S(r, g) \\
\leq & \{23-6 \Theta(\infty ; f)-5 \Theta(\infty ; g)+2 \varepsilon\} T(r)+S(r) .
\end{aligned}
$$

So using Lemma 2.10, we get

$$
(n+1) T(r, f) \leq\{23-6 \Theta(\infty ; f)-5 \Theta(\infty ; g)+2 \varepsilon\} T(r)+S(r) .
$$

In a similar manner, we obtain

$$
(n+1) T(r, g) \leq\{23-5 \Theta(\infty ; f)-6 \Theta(\infty ; g)+2 \varepsilon\} T(r)+S(r) .
$$

From (3.5) and (3.6), we obtain

$$
[n-22+5 \Theta(\infty ; f)+5 \Theta(\infty ; g)+\min \{\Theta(\infty ; f), \Theta(\infty ; g)\}-2 \varepsilon] T(r) \leq S(r)
$$

Since $\varepsilon(>0)$ is arbitrary, (3.7) implies a contradiction. Hence $H \equiv 0$.

Since

$$
\bar{N}\left(r, 0 ; f^{\prime}\right) \leq T\left(r, f^{\prime}\right)-m\left(r, \frac{1}{f^{\prime}}\right) \leq 2 T(r, f)-m\left(r, \frac{1}{f^{\prime}}\right)+S(r, f),
$$

we note that

$$
\begin{aligned}
\bar{N}(r, & \left.; F^{\prime}\right)+\bar{N}\left(r, \infty ; F^{\prime}\right)+\bar{N}\left(r, 0 ; G^{\prime}\right)+\bar{N}\left(r, \infty ; G^{\prime}\right) \\
& \leq \bar{N}(r, 0 ; f)+\bar{N}(r, \infty ; f)+\bar{N}(r, 0 ; g)+\bar{N}(r, \infty ; g)+\bar{N}\left(r, 0 ; f^{\prime}\right)+\bar{N}\left(r, 0 ; g^{\prime}\right) \\
& \leq 4 T(r, f)+4 T(r, g)-m\left(r, 0 ; f^{\prime}\right)-m\left(r, 0 ; g^{\prime}\right)+S(r) \\
& \leq 8 T(r)-m\left(r, 0 ; f^{\prime}\right)-m\left(r, 0 ; g^{\prime}\right)+S(r) .
\end{aligned}
$$


Also using Lemma 2.10, we get

$$
\begin{aligned}
T\left(r, F^{\prime}\right)+m\left(r, \frac{1}{f^{\prime}}\right) & =m\left(r, \frac{f^{n} f^{\prime}}{a}\right)+m\left(r, \frac{1}{f^{\prime}}\right)+N\left(r, \infty ; \frac{f^{n} f^{\prime}}{a}\right) \\
& \geq m\left(r, \frac{f^{n}}{a}\right)+N\left(r, \infty ; f^{n}\right) \\
& =T\left(r, f^{n}\right)+O(1) \\
& =n T(r, f)+O(1) .
\end{aligned}
$$

Similarly

$$
T\left(r, G^{\prime}\right)+m\left(r, \frac{1}{g^{\prime}}\right) \geq n T(r, g)+O(1)
$$

From (3.10) and (3.11), we get

$$
\max \left\{T\left(r, F^{\prime}\right), T\left(r, G^{\prime}\right)\right\} \geq n T(r)-m\left(r, \frac{1}{f^{\prime}}\right)-m\left(r, \frac{1}{g^{\prime}}\right)+O(1)
$$

By (3.9) and (3.12) applying Lemma 2.9, we get either $F^{\prime} \equiv G^{\prime}$ or $F^{\prime} G^{\prime} \equiv 1$.

If $F^{\prime} \equiv G^{\prime}$, then by Lemma 2.12 we obtain $F \equiv G$ or $f \equiv d g$, where $d$ is some $(n+1)$ th root of unity.

If $F^{\prime} G^{\prime} \equiv 1$, then $f^{n} f^{\prime} g^{n} g^{\prime}=a^{2}$. Set $f_{1}=a^{-1 /(n+1)} f$ and $g_{1}=a^{-1 /(n+1)} g$, then $f_{1}^{n} f_{1}^{\prime} g_{1}^{n} g_{1}^{\prime}=$ 1. So using Lemma 2.13, we get $g=c_{1} e^{c z}, f=c_{2} e^{-c z}$, where $c, c_{1}$, and $c_{2}$ are constants and satisfy $\left(c_{1} c_{2}\right)^{n+1} c^{2}=-a^{2}$. This completes the proof of the theorem.

Proof of Theorem 1.6. Let $F=f^{n+1} / a(n+1)$ and $G=g^{n+1} / a(n+1)$. Then $F^{\prime}=f^{n} f^{\prime} / a$ and $G^{\prime}=g^{n} g^{\prime} / a$. Since $f^{n} f^{\prime}$ and $g^{n} g^{\prime}$ share $(a, 1)$, it follows that $F^{\prime}, G^{\prime}$ share $(1,1)$. Suppose that $H \neq \equiv$. Then by Lemma 2.15 , we obtain

$$
\begin{aligned}
T\left(r, F^{\prime}\right) \leq & N_{2}\left(r, 0 ; F^{\prime}\right)+N_{2}\left(r, \infty ; F^{\prime}\right)+N_{2}\left(r, 0 ; G^{\prime}\right)+N_{2}\left(r, \infty ; G^{\prime}\right) \\
& +\frac{1}{2} \bar{N}\left(r, 0 ; F^{\prime}\right)+\frac{1}{2} \bar{N}\left(r, \infty ; F^{\prime}\right)+S\left(r, F^{\prime}\right)+S\left(r, G^{\prime}\right) .
\end{aligned}
$$

We see that

$$
\begin{aligned}
N_{2}\left(r, 0 ; F^{\prime}\right)+N_{2}\left(r, \infty ; F^{\prime}\right) & \leq 2 \bar{N}(r, 0 ; f)+N\left(r, 0 ; f^{\prime}\right)+2 \bar{N}(r, \infty ; f), \\
N_{2}\left(r, 0 ; G^{\prime}\right)+N_{2}\left(r, \infty ; G^{\prime}\right) & \leq 2 \bar{N}(r, 0 ; g)+N\left(r, 0 ; g^{\prime}\right)+2 \bar{N}(r, \infty ; g), \\
\frac{1}{2} \bar{N}\left(r, 0 ; F^{\prime}\right)+\frac{1}{2} \bar{N}\left(r, \infty ; F^{\prime}\right) & \leq \frac{1}{2}\left[\bar{N}(r, 0 ; f)+N\left(r, 0 ; f^{\prime}\right)+\bar{N}(r, \infty ; f)\right] .
\end{aligned}
$$

Again using Lemma 2.10 and proceeding in the same way as done in the proof of Theorem 1.5 , we can show that $S\left(r, F^{\prime}\right)=S(r, f)$ and $S\left(r, G^{\prime}\right)=S(r, g)$. So by Lemmas 2.11 and 2.16, 
we obtain from (3.13) for $\varepsilon>0$ that

$$
\begin{aligned}
T(r, F) \leq & T\left(r, F^{\prime}\right)+N(r, 0 ; f)-N\left(r, 0 ; f^{\prime}\right)+S(r, f) \\
\leq & 2 \bar{N}(r, 0 ; f)+\frac{1}{2} \bar{N}(r, 0 ; f)+\frac{3}{2} N(r, 0 ; f)+2 \bar{N}(r, 0 ; g)+N(r, 0 ; g) \\
& +3 \bar{N}(r, \infty ; f)+3 \bar{N}(r, \infty ; g)+S(r, f)+S(r, g) \\
\leq & (7-3 \Theta(\infty ; f)+\varepsilon) T(r, f)+(6-3 \Theta(\infty ; g)+\varepsilon) T(r, g)+S(r) \\
\leq & \{13-3 \Theta(\infty ; f)-3 \Theta(\infty ; g)+2 \varepsilon\} T(r)+S(r) .
\end{aligned}
$$

So using Lemma 2.10, we get

$$
(n+1) T(r, f) \leq\{13-3 \Theta(\infty ; f)-3 \Theta(\infty ; g)+2 \varepsilon\} T(r)+S(r) .
$$

Similarly, we can obtain

$$
(n+1) T(r, g) \leq\{13-3 \Theta(\infty ; f)-3 \Theta(\infty ; g)+2 \varepsilon\} T(r)+S(r) .
$$

From (3.16) and (3.17), we obtain

$$
[n-12+3 \Theta(\infty ; f)+3 \Theta(\infty ; g)-2 \varepsilon] \leq S(r) .
$$

Since $\varepsilon(>0)$ is arbitrary, we get a contradiction from (3.18). Hence $H \equiv 0$.

Now proceeding in the same way as in the proof of Theorem 1.5 , we obtain either $F^{\prime} \equiv$ $G^{\prime}$ or $F^{\prime} G^{\prime} \equiv 1$. Again proceeding in the same manner as in the proof of Theorem 1.5, we obtain the conclusion of Theorem 1.6. This proves the theorem.

\section{Acknowledgments}

The author is thankful to the unknown referees for valuable suggestions towards the improvement of the paper. The author is also grateful to Dr. I. Lahiri for a technical discussion with him regarding the paper.

\section{References}

[1] T. C. Alzahary and H.-X. Yi, Weighted value sharing and a question of I. Lahiri, Complex Variables Theory Appl. 49 (2004), no. 15, 1063-1078.

[2] W. K. Hayman, Meromorphic Functions, Oxford Mathematical Monographs, Clarendon Press, Oxford, 1964.

[3] I. Lahiri, Value distribution of certain differential polynomials, Int. J. Math. Math. Sci. 28 (2001), no. 2, 83-91.

[4] _ Weighted sharing and uniqueness of meromorphic functions, Nagoya Math. J. 161 (2001), 193-206.

[5] W_ Weighted value sharing and uniqueness of meromorphic functions, Complex Variables Theory Appl. 46 (2001), no. 3, 241-253.

[6] I. Lahiri and A. Banerjee, Weighted sharing of two sets, to appear in Kyungpook Math. J.

[7] I. Lahiri and S. Dewan, Value distribution of the product of a meromorphic function and its derivative, Kodai Math. J. 26 (2003), no. 1, 95-100.

[8] A. Z. Mohon'ko, On the Nevanlinna characteristics of some meromorphic functions, Funct. Anal. Appl. 14 (1971), 83-87. 
[9] R. H. Nevanlinna, Le théorème de Picard-Borel et la théorie des fonctions méromorphes, GauthierVillars, Paris, 1929.

[10] Y. Xu and H. Qu, Entire functions sharing one value IM, Indian J. Pure Appl. Math. 31 (2000), no. 7, 849-855.

[11] C. C. Yang, On deficiencies of differential polynomials. II, Math. Z. 125 (1972), no. 2, 107-112.

[12] C. C. Yang and X. Hua, Uniqueness and value-sharing of meromorphic functions, Ann. Acad. Sci. Fenn. Math. 22 (1997), no. 2, 395-406.

[13] H.-X. Yi, Uniqueness of meromorphic functions and a question of C. C. Yang, Complex Variables Theory Appl. 14 (1990), no. 1-4, 169-176.

[14] Meromorphic functions that share one or two values, Complex Variables Theory Appl. 28 (1995), no. 1, 1-11.

[15] Meromorphic functions that share one or two values. II, Kodai Math. J. 22 (1999), no. 2, 264-272.

[16] H.-X. Yi and L.-Z. Yang, Meromorphic functions that share two sets, Kodai Math. J. 20 (1997), no. 2, 127-134.

Abhijit Banerjee: Department of Mathematics, Kalyani Government Engineering College, West Bengal 741235, India

E-mail address: a.banerjee@hotpop.com 


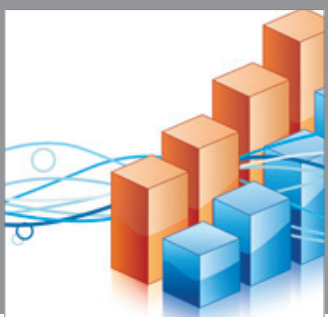

Advances in

Operations Research

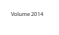

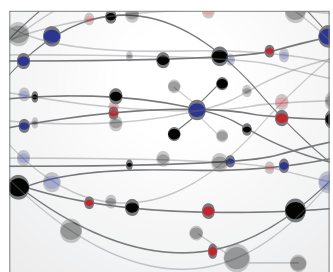

\section{The Scientific} World Journal
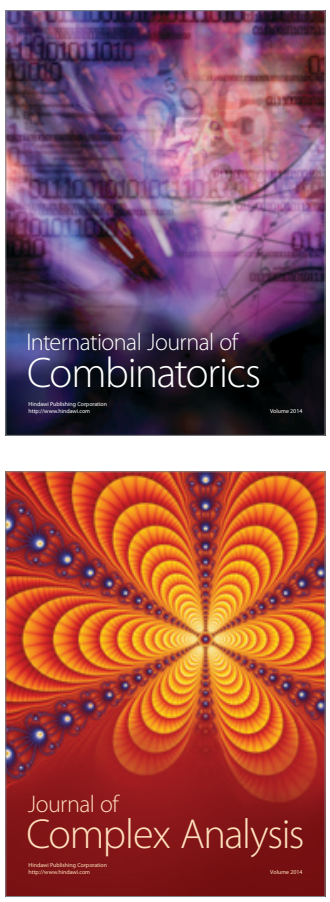

International Journal of

Mathematics and

Mathematical

Sciences
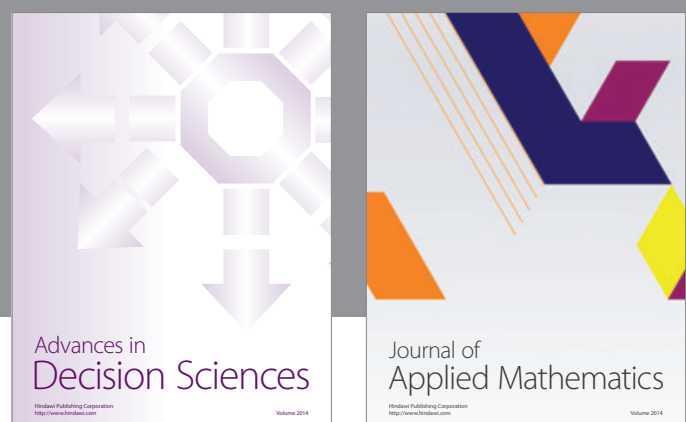

Journal of

Applied Mathematics
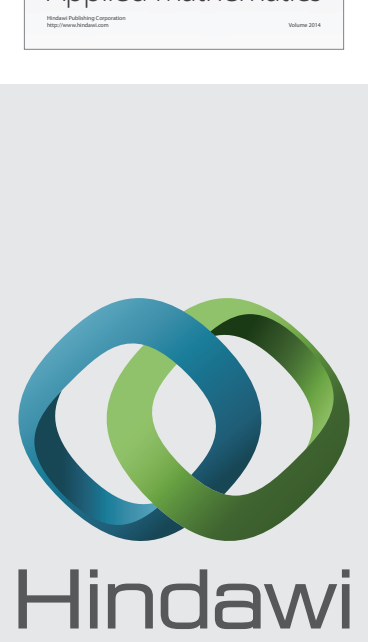

Submit your manuscripts at http://www.hindawi.com
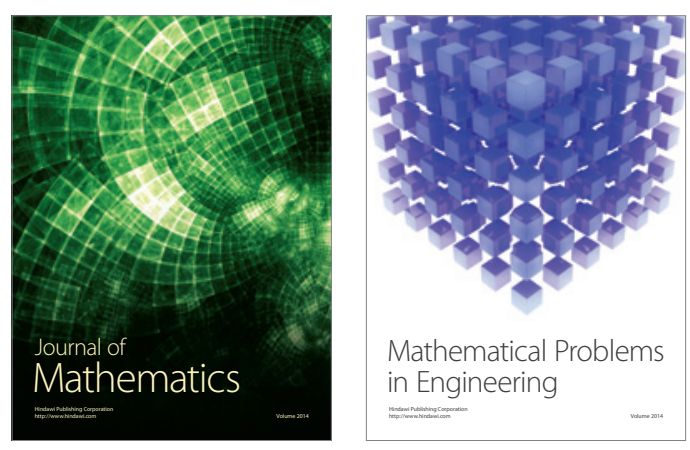

Mathematical Problems in Engineering
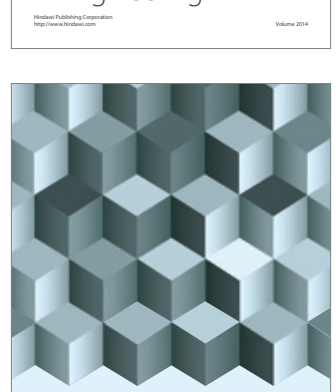

Journal of

Function Spaces
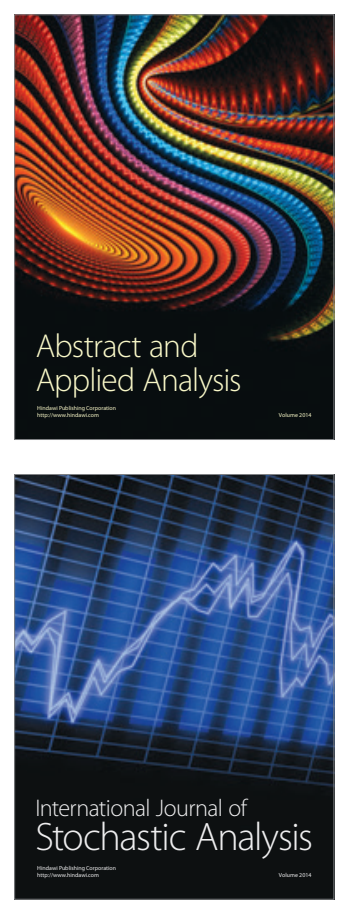

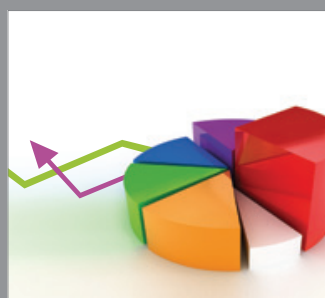

ournal of

Probability and Statistics

Promensencen
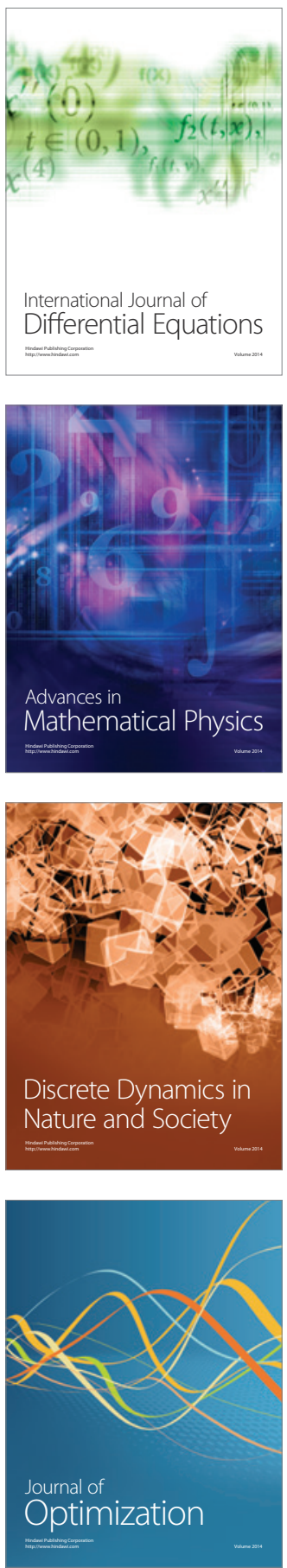